\title{
Administrative Gender Code
}

National Cancer Institute

\section{Source}

National Cancer Institute. Administrative Gender Code. NCI Thesaurus. Code 693501.

A coded value specifying the physical or societal properties by which male is distinguished from female. 